\title{
Avaliação da assistência ao parto no norte do Paraná pelo Índice de Bologna
}

\author{
Assessment of childbirth care in northern Paraná by the Bologna Index
}

\author{
Evaluación de la atención al parto en el norte de Paraná por el Índice de Bolonia
}

Emily Marques Alves ${ }^{1 *}$, Nathalia Jung Ferreira Malta ${ }^{1}$, Pamela Panas dos Santos Oliveira ${ }^{1}$, Lethicia Scheller Oliveira ${ }^{1}$, Keli Regiane Tomeleri da Fonseca Pinto ${ }^{1}$, Rosângela Aparecida Pimenta Ferrari ${ }^{1}$, Catia Campaner Ferrari Bernardy ${ }^{1}$.

\section{RESUMO}

Objetivo: Este estudo tem como objetivo avaliar a assistência ao parto em maternidades do norte do Paraná pelo Índice de Bologna. Métodos: Pesquisa de caráter quantitativo e transversal desenvolvido em quatro maternidades, contemplando 417 mulheres entre o período de janeiro e junho de 2017. O índice de Bologna avalia a assistência prestada no parto normal por meio de cinco variáveis: presença de um acompanhante; uso do partograma; ausência de estimulação ou indução, uso de instrumental fórceps/vácuo; parto na posição não supina; contato pele a pele. Cada uma pode pontuar 0 , caso não esteja presente, ou 1, caso a variável esteja presente. Para avaliação do parto normal foi incluído 229 mulheres. Resultados: A pontuação do Índice de Bologna foi respectivamente, 1 ponto (4\%), 2 pontos $(42 \%), 3$ pontos $(44,4 \%), 4$ pontos $(9,2 \%)$ e 5 pontos $(0,4 \%)$. Destacou-se como positivo a utilização do partograma durante o trabalho de parto, e negativo o uso da posição supina na maior parte dos partos. Conclusão: Apesar da prevalência de uma pontuação mediana (3 pontos), foi possível concluir que durante a assistência há ocorrência de práticas não recomendadas, que trazem prejuízos a mulher. Por fim reforça-se a importância do Índice de Bologna para avaliação da assistência.

Palavras-chave: Parto obstétrico, Parto humanizado, Assistência ao parto, Pesquisa sobre serviços de saúde, Saúde da mulher.

\begin{abstract}
Objective: This study aims to evaluate childbirth assistance in maternity hospitals in northern Paraná using the Bologna Index. Methods: A quantitative and cross-sectional research carried out in four maternity hospitals, covering 417 women between the period of January and June 2017. The Bologna index evaluates the assistance provided in normal childbirth through five variables: presence of a companion; use of the partogram; absence of stimulation or induction, use of forceps / vacuum instruments; delivery in the non-supine position; skin to skin contact. Each one can score 0, if it is not present, or 1, if the variable is present. For the evaluation of normal delivery, 229 women were included. Results: The Bologna Index score was, respectively, 1 point $(4 \%), 2$ points (42\%), 3 points $(44.4 \%), 4$ points $(9.2 \%)$ and 5 points $(0.4 \%)$. The use of the partograph during labor was highlighted as positive, and the use of the supine position in most births was negative. Conclusion: Despite the prevalence of a median score (3 points), it was possible to conclude that during assistance there are non-recommended practices, which bring harm to women. Finally, the importance of the Bologna Index for the evaluation of assistance is reinforced.
\end{abstract}

Keywords: Obstetric childbirth, Humanized birth, Childbirth assistance, Health services research, Women's health.

\section{RESUMEN}

Objetivo: Este estudio tiene como objetivo evaluar la asistencia al parto en las maternidades del norte de Paraná utilizando el Índice de Bolonia. Métodos: Investigación cuantitativa y transversal realizada en cuatro maternidades, que abarcó a 417 mujeres entre el período de enero a junio de 2017. El índice de Bolonia evalúa la asistencia prestada en el parto normal a través de cinco variables: presencia de un acompañante; uso del partograma; ausencia de estimulación o inducción, uso de fórceps / instrumentos de vacío; parto en

1 Universidade Estadual de Londrina, Londrina - PR. *E-mail: marquesalvesemily@gmail.com 
posición no supina; contacto piel a piel. Cada uno puede puntuar 0 , si no está presente, o 1, si la variable está presente. Para la evaluación del parto normal, se incluyeron 229 mujeres. Resultados: La puntuación del Índice de Bolonia fue, respectivamente, 1 punto (4\%), 2 puntos (42\%), 3 puntos (44,4\%), 4 puntos (9,2\%) y 5 puntos $(0,4 \%)$. El uso del partograma durante el trabajo de parto se destacó como positivo y el uso de la posición supina en la mayoría de los partos fue negativo. Conclusión: A pesar de la prevalencia de una puntuación mediana (3 puntos), se pudo concluir que durante la asistencia existen prácticas no recomendadas, que son perjudiciales para las mujeres. Finalmente, se refuerza la importancia del Índice de Bolonia para la evaluación de la ayuda.

Palabras clave: Parto obstétrico, Nacimiento humanizado, Asistencia para el parto, Investigación de servicios de salud, La salud de la mujer.

\section{INTRODUÇÃO}

O momento de parir vem se tornando gradativamente mais medicalizado e envolvido por uma assistência intervencionista. Um momento que deveria ser fisiológico e único na vida da mulher, acaba sendo permeado por muitos medos e inseguranças (KOTTWITZ F, et al., 2018). Por isso ações que objetivam o aumento da qualidade em relação a assistência de saúde em obstetrícia são fundamentais para redução da mortalidade materna. Ações humanizadas e o combate aos diferentes tipos de abusos obstétricos, contribuem para segurança e bem-estar materno e infantil (SOUZA JP, 2018).

A mortalidade materna é considerada óbito ocorridos no período gestacional ou até 42 dias após o parto, independente das intercorrências gestacionais que levaram há este desfecho. Sendo dividida em causas direta e indireta. As causas diretas são aquelas resultantes de complicações obstétricas no período gravídicopuerperal resultante da falha na assistência e/ou omissão de tratamento, já as causas indiretas são aquelas originadas de patologias pré-existentes ou desenvolvidas no período gestacional levando ao desfecho negativo da gestação (SCHOLZE AR, et al., 2021)

Dessa forma a redução da mortalidade materna foi um dos Objetivos do Milênio a serem cumpridos até o ano de 2015, e apesar de o objetivo não ter sido atingindo por completo, estima-se que 1,5 milhões de mortes foram evitadas no Brasil e no mundo. Atualmente ainda segue como uma das metas dos Objetivos de Desenvolvimento Sustentável, que visa a redução para menos de 70 mortes maternas por 100.000 nascidos vivos até 2030, tendo em vista que no ano de 2019 ocorreu 1.576 morte materna (SOUZA JP, 2018, DEPRTAMENTO DE INFROMATICA DO SUS (DATASUS), 2021).

No cenário brasileiro, um marco na história da Assistência à Saúde da Mulher, foi a criação, em 1984, do Programa de Assistência Integral à Saúde da Mulher, primeira política que, mesmo indiretamente, iniciou a humanização no cuidado à mulher, e visava a integralidade e a autonomia feminina quanto ao seu corpo (APOLINÁRIO D, et al., 2016).

O Ministério da Saúde, no ano de 2000, criou o Programa de Humanização do Pré-natal e Nascimento, surgindo de uma necessidade de atenção específica à gestante, ao recém-nascido e à mulher no puerpério. Ficou estabelecido por meio deste Programa que os serviços de saúde deveriam garantir às mulheres consultas de pré-natal e exames estabelecidos, realização de práticas educativas, e atendimento de qualidade em hospitais (ROMÃO RS, et al., 2018).

Em 2011 o Governo Federal criou o programa Rede Cegonha, que assegura a gestantes usuárias do Sistema Único de Saúde, atendimento humanizado, qualificado, com direito ao planejamento reprodutivo, à gestação, ao parto e até ao segundo ano de vida da criança (UNIVERSIDADE FEDERAL DO MARANHÃO, 2015).

No cenário mundial, em 1996, a Organização Mundial da Saúde (OMS) lançou um conjunto de recomendações presentes no documento Boas Práticas de Atenção ao Parto e Nascimento, que classificava algumas práticas como úteis e que deveriam ser estimuladas, outras práticas que deviam ser eliminadas por serem prejudiciais, além das práticas que devem ser utilizadas com cautela até comprovações de novas pesquisas (WHO, 1996). 
A avaliação da assistência à saúde tem sido considerada como forte ferramenta para o planejamento da gestão do sistema de saúde, possibilitando melhorias no espaço de trabalho, durante as tomadas de decisões. Os indicadores são parte importe desse processo avaliativo no serviço de saúde pois permite a descrição de determinado fatores buscado medidas estratégicas com base na população estudada (CARVALOR I DA S e BRITO RS, 2016).

Diante de todas as discussões sobre importância da realização dos indicadores como fonte de dados e qualificação na assistência prestada ao binômio mãe-filho, a OMS criou alguns indicadores que avaliam de forma quantitativa se a atenção ao parto normal está sendo efetiva e humanizada. Assim, em uma reunião intitulada Força Tarefa Perinatal, na cidade de Bologna, no ano de 2000, foi estabelecido pela OMS o Índice de Bologna, com objetivo de avaliar como o Trabalho de Parto é manejado dentro de uma instituição de saúde, por meio de algumas variáveis (CHALMERS B e POTER R, 200; GIGLIO MRO, et al., 2011).

As cinco variáveis do Índice de Bologna que devem ser avaliadas no decorrer do trabalho de parto, são a presença do acompanhante durante o parto; presença de partograma; ausência de estimulação do trabalho de parto, como o uso de ocitocina, manobra de Kristeller, episiotomia, ou parto instrumental (fórceps/vácuo extrator) e cesariana de emergência; parto na posição não supina e contato pele a pele da mãe com o recémnascido (ROMÃO RS, et al., 2018; OLIVEIRA FAM, et al., 2015).

Para a OMS, uma assistência ao trabalho de parto e nascimento é adequadamente avaliada quando se realiza a análise de algumas varáveis, utilizando-se o Índice de Bologna denominado como indicador C, associado aos indicadores "porcentagem de mulheres submetidas ao trabalho de parto induzido/submetidas à cesárea eletiva" (indicador B) e "porcentagem de mulheres atendidas por profissionais de saúde especialistas" (indicador A) (WHO, 1996; OLIVEIRA FAM, et al., 2015).

Desta forma, este estudo tem como objetivo avaliar a assistência ao parto em maternidades do norte do Paraná pelo Índice de Bologna.

\section{MÉTODOS}

Trata-se de uma pesquisa de caráter quantitativo, descritivo, transversal e prospectivo. Os dados foram tabulados por meio do Microsoft Office-Excel ${ }^{\circledR}$ e analisados no programa Statistical Package for the Social Science (SPSS) e versão 20.0, utilizou-se o Teste Qui-quadrado $(p \leq 0,05)$ e por meio de valores absolutos e medidas de frequência relativa. As variáveis dependentes foram avaliadas por meio do teste de regressão binária logística, para avaliação da razão de chances (RC) e intervalo de confiança (IC) de 95\% (IC 95\%), considerando os resultados estatisticamente não significativos.

Esta estudo é um recorte da pesquisa multicêntrica intitulada: "Rede Mãe Paranaense na Perspectiva da Usuária: o Cuidado da Mulher no Pré-natal, Parto, Puerpério e da Criança" que foi desenvolvida em municípios de três Regionais de Saúde (RS): 9aㅡ RS de Foz do Iguaçu, 10ª RS de Cascavel e 17ª RS de Londrina, Paraná, região Sul do Brasil, para analisar a assistência materna no pré-natal, parto e puerpério e o seguimento da criança, após a Implantação da Rede Mãe Paranaense na perspectiva da mulher/usuária, nos primeiros dezoito meses de vida da criança e/ou pós-parto.

O estudo multicêntrico foi composto de 417 mulheres, porém, para esta análise foram incluídas 229 mulheres que pariram em quatro maternidades de pequeno e médio porte, que atendem gestação de risco habitual e intermediário nas cidades de Londrina, Cambé, Rolândia e Ibiporã, pertencentes à $17^{\text {a }}$ Regional de Saúde (RS).

A coleta dos dados foi realizada no momento do parto, entre janeiro e junho de 2017 . Os critérios de inclusão foram mulheres que realizaram o parto nas maternidades em estudo, bem como não apresentaram nenhum tipo de agravo e/ou problema de saúde que impediria a sua participação. Os critérios de exclusão foram mulheres que apresentaram parto de óbito fetal, ou mulheres graves e intubadas com nível de consciência prejudicado.

As fontes de dados utilizadas foram a Carteira de Saúde da Gestante, os prontuários e entrevista com as puérperas nas maternidades. Foram analisadas questões referentes a caracterização sociodemográfica e 
econômica, as variáveis do índice de Bologna, índice de parto induzido, cesáreas eletivas, e mulheres que foram atendidas por profissionais de saúde especializados.

O índice de Bologna avalia a assistência prestada às mulheres no momento do parto normal por meio de cinco variáveis: presença de um acompanhante; uso do Partograma; ausência de estimulação ou indução (inclui a ocitocina, manobra de Kristeller e episiotomia) uso de instrumental fórceps/vácuo; parto na posição não supina; contato pele a pele por pelo menos 30 minutos. Cada variável pontua um (1) se estiver presente, e zero (0) se estiver ausente. O valor do índice é obtido por meio da somatória dos resultados. O valor zero (0) indica uma má assistência ao parto, de um (1) ao quatro (4) indica assistência intermediaria e cinco (5), indica uma boa qualidade na assistência ao parto. Dessa forma o mesmo foi aplicado apenas nas fichas relacionadas aos partos vaginais ocorridos nas maternidades das $17^{\circ} \mathrm{RS} / \mathrm{PR}$.

Todos os participantes receberam as informações do estudo de forma verbal e por escrito. O Termo de Consentimento Livre e Esclarecido foi assinado pela pesquisadora e pelos participantes em duas vias, sendo uma elas entregue aos participantes. Por estar inserido na pesquisa multicêntrica, a pesquisa foi aprovada pelo Comitê de Ética da Universidade Estadual de Londrina (CEP/UEL) sob o Parecer: 2.053.304.

\section{RESULTADOS}

A população estuda foi composta por 250 mulheres, $71,6 \%$ (179) tinham idade entre 20 e 34 anos, 56,0\% (140) se consideravam brancas, $85,6 \%$ (214) apresentavam companheiro, 52,8\% (132) tinham ensino médio completo e ensino superior. Com relação a paridade 94 (38\%) apresentaram nenhuma gestação anterior.

Para a aplicação do Índice de Bologna (Indicador C), que avalia a adequação da assistência ao parto normal, foram analisados os 229 partos vaginais e excluíram-se as cesáreas, e as categorias utilizados foram Presença de um acompanhante; Uso do partograma; Ausência de estimulação; Parto na posição não supina e Contato pele a pele.

O Índice de Bologna variou entre um (1) a cinco (5) pontos, sendo que apenas uma mulher teve boa assistência ao parto, e 99,6\% (228) das mulheres apresentaram assistência intermediaria ao parto. Entretanto, ressalta-se que nenhuma maternidade teve pontuação zero (Tabela 1).

Tabela 1 - Pontuação do Índice de Bologna, aplicado aos partos vaginais realizados nas maternidades da 17a Regional de Saúde, entre janeiro a junho de 2017.

\begin{tabular}{ccc}
\hline VARIÁVEIS & POPULAÇÃO & PORCENTAGEM (\%) \\
\hline Parto Vaginal & 229 & $55 \%$ \\
0 ponto & 0 & $0 \%$ \\
1 ponto & 9 & $4 \%$ \\
2 pontos & 96 & $42 \%$ \\
3 pontos & 102 & $44,4 \%$ \\
4 pontos & 21 & $9,2 \%$ \\
5 pontos & 1 & $0,4 \%$ \\
\hline
\end{tabular}

Fonte: Alves EM, et al., 2021.

Foi realizado análise estatística com o indicador $C$ (índice de Bologna) e as variáveis sociodemográfica (idade, escolaridade), obstétrica (número de gestação, tipo de parto) e variáveis relacionado ao indicador $\mathrm{A}$ e B. No memento da analise não houve associação estatísticas entre as variáveis descrias s acima, por eSte motivo houve necessidade apenas da avaliação da frequência, obtendo um dado descritivo do indicado A, B e C.

Quanto a presença do acompanhante de livre escolha da mulher 93,2\% (233) obteve acompanhante durante o momento do parto. Relacionado ao uso do partograma $92 \%$ (230) dos nascimentos foram usados essa ficha para o registro da evolução do tralho de parto (Tabela 2). 
Com relação a ausência do uso de estimuladores, 58,0\% (147) não fizeram uso de ocitocina e com relação ao uso do misoprostol $80,0 \%$ (200) não houve a presença desta conduta. Dentre as medidas mais utilizadas durante a assistência ao parto foi o uso de ocitocina 35,6\% (89), seguida de $6,0 \%$ (15) de realizações de epsiotomia, manobra de Kristeller 10,4\% (26) e parto instrumental apenas 0,8\% (2) (Tabela 2).

Relacionado ao parto em posição não supina somente 5,6\% (14) tiveram seus partos em posição de cócoras, vertical, lateral ou em quatro apoios, sendo que 79,2\% (198) das mulheres tiveram seus partos em posição litotômica. Tratando do estabelecimento do contato pele a pele imediatamente após o parto menos da metade $32,0 \%(80)$ realizaram pelo menos $30 \mathrm{mim}$ de contato pele a pele conforme a orientação da OMS (Tabela 2).

Tabela 2- Variáveis do Índice de Bologna, aplicadas nos partos vaginais realizados nas maternidades da 17ª Regional de Saúde, entre janeiro a junho de 2017.

\begin{tabular}{ccc}
\hline VARIÁVEIS & POPULAÇÃO & PORCENTAGEM (\%) \\
\hline Presença de acompanhante & 213 & $93 \%$ \\
Uso do partograma & 224 & $98 \%$ \\
Ausência de estimulação & 65 & $28,4 \%$ \\
Parto na posição não supina & 9 & $3,8 \%$ \\
Contato pele a pele & 81 & $35,4 \%$ \\
\hline
\end{tabular}

Fonte: Alves EM, et al., 2021.

Em relação ao indicador $A$, que avalia mulheres atendidas por profissionais de saúde especializado, a maior parte destas mulheres $59,6 \%$ (149) foi atendida por um médico, enquanto $35,2 \%$ (88) tiveram seus partos assistidos por enfermeiros obstetras.

O indicador $B$ que se refere à cesárea eletiva ou ao trabalho de parto induzido, destaca que $9,6 \%(24)$ das mulheres foram submetidas à cesariana, 4,0\% (10) que foi realizado cesárea por urgência e emergência e seguida $3,6 \%$ (9) que foram interativas.

\section{DISCUSSÃO}

Para a OMS, uma assistência ao trabalho de parto e nascimento é adequadamente avaliada quando se realiza a análise conjunta dos indicadores $A$. B e C, obtendo-se uma qualidade máxima de assistência ao parto quando se encontra associação de baixas porcentagens de cesarianas eletivas e/ou induções de parto, assim como altas porcentagens de partos acompanhados por profissional de saúde e alto Escore de Bologna (WHO, 1996; OLIVEIRA FAM, et al., 2015).

Neste estudo não foi encontrado associação estatística entre as variáveis do índice de Bologna e os indicadores A e B assim como para os dados sociodemográfico, nos fazendo questionar o que determina a qualidade da assistência prestada para as mulheres que usam as maternidades de risco habitual e intermediário. Entretanto vale ressaltar que ao analisar o índice de Bologna percebemos que o score predominante foi aquele referente a qualidade intermediária.

Deve destacar que entre as cinco regiões do Brasil o Paraná tem a menor taxa de mortalidade, sabe-se que essa taxa é um importante indicador de saúde que reflete a qualidade da atenção prestada à mulher $\mathrm{e}$ quando está elevada pode indicar uma prestação de serviço inadequada a esse grupo (DEPRTAMENTO DE INFROMATICA DO SUS - DATASUS, 2021).

Em estudo desenvolvido em uma maternidade na cidade de Uberlândia, com amostra de 82 gestantes, identificou um escore Bologna com pontuações três (47\%) (ROMÃO RS, et al., 2018). Outro estudo realizado em três maternidades em Curitiba identificou índices Bologna baixos, variando o escore de um ao três. Estes estudos foram compatíveis com os resultados encontrados nessa pesquisa, demostrado a necessidade de implementação de medidas para a capacitação e o fortalecimento da prática baseada em evidência e estudo qualitativos para a identificação de ideias subjetivas dos profissionais e usuárias desse serviço. 
Em relação a assistência prestada no trabalho de parto, a figura do médico prevaleceu sobre os atendimentos. Realidade empregada de forma diferente em uma análise realizada em dois hospitaismaternidade da rede pública municipal do Rio de Janeiro, onde a maior parte dos partos foram acompanhados por enfermeiras obstétricas, 68,5\% e 43\%, respectivamente (VARGENS OMC, et al., 2017).

O Ministério da Saúde incentiva a inclusão de outros profissionais na equipe de assistência ao parto, como enfermeiros obstetras, obstetrizes, doulas, fisioterapeutas entre outros, proporcionando uma assistência integral a saúde da mulher. Incentiva, principalmente, nesse cenário, a inclusão do enfermeiro obstetra na realização de partos de risco habitual, promovendo a ampliação do cuidado e assistência humanizada (BRASIL, 2014). Este reforça a importância de se implementar práticas baseadas em evidências, assim como, oferecer uma assistência humanizada no momento do parto e nascimento tendo em vista que o parto e um evento familiar e permeados de sentimentos e emoções (BRASIL, 2015).

O resultado positivo obtido neste estudo assemelha-se ao de uma maternidade em Belo Horizonte, onde $95,4 \%$ dos partos contaram com acompanhante, visando à assistência respeitosa e humanizada (SOUSA AMM, et al., 2016). Tendo em vista que a presença do acompanhante é regulamentada por lei onde a mulher tem o direito de escolher um acompanhante desde o momento da internação até a sua alta, tanto para os serviços públicos como para os privados (BRASIL, 2005).

A OMS instituiu a utilização do partograma em 1996 pelas maternidades com o objetivo de detectar precocemente o risco gestacional pelos profissionais capacitados para atuar no processo do parto. $O$ partograma esteve presente na grande maioria dos partos deste estudo, favorecendo a identificação de possíveis distocias durante o parto e nascimento e contribuído para desfechos positivos (OLIVEIRA FAM, et al., 2015).

A maior frequência de estimulação encontrada nesta pesquisa foi o uso da ocitocina para indução do trabalho de parto. Mesmo sendo contraindicado o uso rotineiro desta prática pela OMS, devido a possibilidade de causar danos a mãe e ao bebê, quando usada de forma indevida, ainda se observa grande utilização em diversas maternidades brasileiras (SOUSA AMM, et al., 2016; GUIDA NFB, et al., 2017).

A episiotomia é uma intervenção que permanece sendo utilizada nas instituições de saúde (APOLINÁRIO D, et al., 2016; GUIDA NFB, et al., 2017), apesar dos estudos científicos evidenciarem que esse procedimento não cumpre os objetivos que justificariam sua realização, como por exemplo, o efeito protetor sobre o períneo. Embora a OMS aconselhe que a realização da episiotomia não ultrapasse $10 \%$ nas a maternidades, as evidências científicas não justificam sua execução de forma rotineira (APOLINÁRIO D, et al., 2016; SOUSA AMM, et al., 2016).

O uso da manobra de Kristeller nas maternidades avaliadas, mesmo em pequena quantidade, ainda esteve presente, como também em outras maternidades (SOUSA, et al., 2016; GUIDA, et al., 2017). A manobra é considerada perigosa no meio obstétrico, pode causar riscos para as mulheres e seus bebês, sendo uma prática não recomendada em muitos países (GAMA SGN, et al., 2016; SOUSA AMM, et al., 2016).

Em relação à posição adotada no trabalho de parto, houve o predomínio da posição não vertical (SOUSA AMM, et al., 2016), prática que deve ser desencorajada, preferindo posições verticalizadas. As posições verticais são consideradas mais fisiológicas e benéficas ao binômio, oferecendo maior conforto e autonomia no momento do nascimento. Realidade abordada de maneira diferente em uma maternidade pública do Rio de Janeiro, onde $65,5 \%$ das gestantes realizam parto na posição vertical, prática essa influenciada pelas enfermeiras obstétricas (VARGENS OMC, et al., 2017).

Observou-se que o contato pele a pele foi pouco explorado pelas maternidades estudadas nesta pesquisa. Um pouco mais da metade das mulheres não realizou o contato pele a pele de forma que é orientada pela OMS. A recomendação e que seja realizado o contato pele a pele do recém-nascido ativo imediatamente após o parto, por pelo menos 30 minutos. Esta prática é realizada com o intuito de prevenir hipotermia no neonato, favorecer o vínculo entre mãe e filho, além da estimulação precoce ao aleitamento (OLIVEIRA FAM, et al. 2015). Estudos realizados em maternidades Brasileiras apontam resultados positivos nessa prática, com $75,1 \%$ e $84 \%$ de contato pele a pele após o parto (APOLINÁRIO D, et al., 2016; OLIVEIRA FAM, et al., 2015). 
O Índice de Bologna associado aos indicadores A e B auxilia na construção de instrumentos para a monitorização da assistência obstétrica realizada ao binômio mãe-filho. Devendo ser incentivado e adotado nos serviços de saúde, a fim de estimar de forma simples e prática a qualidade do processo de assistência ao parto. Outras práticas evidenciam necessidade de aprimoramento e incentivo, a fim de se alcançar um parto humanizado e de excelência, com respeito a mulher e ao recém-nascido (MOURA NAS, et al., 2020).

\section{CONCLUSÃO}

A partir da aplicação do Índice de Bologna foi possível estabelecer um panorama das quatro maternidades da $17^{\text {a }}$ Regional de Saúde, proporcionando assim o reconhecimento da assistência prestada às parturientes e as limitações presentes nos serviços, proporciona a ampliação do olhar sobre a assistência prestada às mulheres que usam as maternidades como referência para parto em busca do melhor desfecho para a gestação. Assim, os serviços podem estabelecer melhores estratégias visando a excelência do cuidado e proporcionando ambiente adequado para o nascimento, seguindo as recomendações da Organização Mundial da Saúde.

\section{REFERÊNCIAS}

1. APOLINÁRIO D, et al. Practices in delivery and birth care from mothers' perspective. Rev Rene. 2016; 17(1): 20-8;

2. BRASIL. Lei n 11.108, de 7 de abril de 2005. Garante às parturientes o direito à presença de acompanhante durante o trabalho de parto, parto e pós-parto imediato, no âmbito do Sistema Único de Saúde - SUS. Diário Oficial da União. Brasilia; 2005;

3. BRASIL. Ministério da Saúde. Cadernos humaniza SUS. Humanização do parto e do nascimento. Brasília (DF): $\begin{array}{llll}\text { Ministério da } & \text { Saúde; } & \text { Disponível em: }\end{array}$ https://www.redehumanizasus.net/sites/default/files/caderno_humanizasus_v4_humanizacao_parto.pdf;

4. BRASIL. Ministério da Saúde. Diretrizes de Atenção à Gestante: a operação cesariana. Brasília (DF): Ministério da Saúde; 2015. Disponível em: http://conitec.gov.br/images/Relatorios/2016/Relatorio_Diretrizes-Cesariana_final.pdf;

5. CALDEIRA S, et al. Nursing care actions in the paranaense mother network program. Rev Min Enferm 2017; 21(e992): 1-9;

6. CHALMERS B, PORTER R. Assessing effective care in normal labor: The Bologna Score. Birth. 2001; 28(2): 79-83;

7. DEPRTAMENTO DE INFROMATICA DO SUS (DATASUS). Informação de saúde, Epidemiológicas e Morbidade: banco de dados, 2018. Disponível em: http://tabnet.datasus.gov.br/cgi/deftohtm.exe?sim/cnv/mat10pr.def.

8. GAMA SGN, et al. Labor and birth care by nurse with midwifery skills in Brazil. Reproduct Health 2016; 13(Suppl 1): 123;

9. GUIDA NFB, et al. Compliance of nursing care practices with technical recommendations for normal birth. Rev Rene 2017; 18(4): 543-50.

10. KOTTWITZ F, et al. Route of birth delivery preferred by mothers and their motivations. Rev Esc Anna Nery. 2018; 22(1): 1-8;

11. MOURA NAS, et al. Análise de prática na asssitência ao parto e pós-parto hospitalar. Revista Rene 2020, 21: e43671;

12. OLIVEIRA FAM, et al. The use of Bologna Score to assess normal labor care in maternities. Rev Gaúcha Enferm. 2015; 36(spe): 177-18;

13. RIBEIRO JF, et al. Evaluation of normal delivery service at a public motherhood. Revista Eletrônica Gestão \& Saúde 2016, 7(1): 65-81;

14. ROMÃO RS, et al. Quality of obstetric assistance related to vaginal delivery: a cross-sectional study. Rev Enferm Centro-O Min. 2018; 8: e2907;

15. SCHOLZE AR, et al. Mortalidade maternal: compartivo após implantação da Rede Mãe Paranaense. Revista Journal of nursing and health, 2020; 10(2): e20102007;

16. SOUSA AMM, et al. Practices in childbirth care in maternity with inclusion of obstetric nurses in Belo Horizonte, Minas Gerais. Esc Anna Nery 2016; 20(2): 324-31;

17. SOUZA JP. Maternal mortality and the new objectives of sustainable development (2016-2030). Rev Bras Ginecol Obstet 2018; 37(12): 549-51;

18. UNIVERSIDADE FEDERAL DO MARANHÃO. UNA-SUS. Redes de atenção à saúde: a Rede Cegonha. São Luís; 2015;

19. VARGENS OMC, et al. The contribution of nurse midwives to consolidating humanized childbirth in maternity hospitals in Rio de Janeiro-Brazil. Esc Anna Nery. 2017; 21(1): e-20170015;

20. WORLD HEALTH ORGANIZATION (WHO). Maternal and Newborn Health/Safe Motherhood Unit. Care in normal birth: a practical guide. Geneva: WHO/FRH/MSM; 1996. 